\title{
Diagnostic value of NSE factor combined with ultrasound hemodynamic indexes in cervical lymph node metastasis of lung cancer
}

\author{
YANSONG LI ${ }^{1}$, YONG KUANG ${ }^{2}$, YANZHEN JIA ${ }^{2}$ and SHUFANG BAI ${ }^{3}$ \\ ${ }^{1}$ Department of Ultrasound, Qingdao Municipal Hospital (Group), Qingdao, Shandong 266011; \\ ${ }^{2}$ Department of Physical Diagnostics, Qingdao Ninth People's Hospital, Qingdao Municipal Hospital (Group), Qingdao, \\ Shandong 266002; ${ }^{3}$ Department of Ultrasound, The People's Hospital of Zhangqiu Area, Jinan, Shandong 250200, P.R. China
}

Received May 20, 2019; Accepted September 30, 2019

DOI: $10.3892 / \mathrm{ol} .2020 .11621$

\begin{abstract}
Value of neuron-specific enolase (NSE) factor combined with ultrasound hemodynamic parameters in the diagnosis of cervical lymph node metastasis of lung cancer was explored. The clinical data of 85 patients with lung cancer, admitted to Qingdao Municipal Hospital (Group) from January 2015 to December 2016, were retrospectively analyzed. According to the results of pathological examination, 47 patients with cervical lymph node metastasis were enrolled in the metastatic group and 38 patients without lymph node metastasis were enrolled in the non-metastatic group. The expression level of NSE in serum and the hemodynamic indicators of blood flow resistance index (RI) and pulsatility index (PI) were compared between the two groups. ROC curve analysis was used to analyze the diagnostic efficacy of NSE, RI, PI, and their combination in lymph node metastasis of lung cancer. The NSE, RI and PI indexes in the metastatic group were significantly higher than those in the non-metastatic group $(\mathrm{P}<0.05)$. The sensitivity and specificity of NSE in the diagnosis of cervical lymph node metastasis of lung cancer were 73.68 and $72.34 \%$, respectively; the sensitivity and specificity of RI were 78.95 and $80.85 \%$, respectively; the sensitivity and specificity of PI were 81.58 and $68.09 \%$, respectively. Also, the sensitivity and specificity of NSE combined with RI were 89.47 and $61.70 \%$, respectively, and the diagnostic AUC was 0.881 . The sensitivity and specificity of NSE combined with PI were 92.11 and $74.47 \%$, respectively, and the diagnostic AUC was 0.905. NSE, RI, and PI have certain diagnostic value for cervical lymph node metastasis of lung cancer, however, the combined diagnosis is more valuable,
\end{abstract}

Correspondence to: Dr Yansong Li, Department of Ultrasound, Qingdao Municipal Hospital (Group), 1 Jiaozhou Road, Qingdao, Shandong 266011, P.R. China

E-mail: songfu96682153@126.com

Key words: neuron-specific enolase, blood flow resistance index, blood flow pulsation index, lung cancer, lymph node metastasis and can be used as the auxiliary diagnosis of cervical lymph node metastasis of lung cancer.

\section{Introduction}

Lung cancer is one of the most common malignant tumors in clinic, and because its early symptoms are not obvious, a number of patients are diagnosed at late stages, which is one of the reasons leading to a low survival rate within 5 years. Numerous related investigations have shown that lung cancer has the highest clinical mortality rate at present $(1,2)$. Besides, the metastasis and recurrence of lung cancer is also one of the main causes of poor prognosis and low survival rate in patients with lung cancer (3). Lymph node metastasis is one of the most common diffusion pathways in lung cancer, and one of the main causes of death among patients with lung cancer (4). Therefore, the accurate diagnosis of lymph node metastasis in patients with lung cancer has an important clinical significance for the timely and effective treatment, and the improvement of survival rate of patients with lung cancer.

Serum tumor markers play an important role in the diagnosis of lung cancer. Neuron-specific enolase (NSE) is one of the most important tumor markers in the clinical diagnosis of lung cancer (5). NSE is a substance of the acidic protease-containing neuronal tissues, such as the neuroendocrine and the peripheral nerve tissues. Since $80 \%$ of lung cancers are tumors of neuroendocrine origin, NSE is also one of the tumor markers with high sensitivity to the diagnosis of lung cancer (6). However, its single detection has some limitations to the detection of lymph node recurrence and metastasis in lung cancer patients. Therefore, in the present study, its combination with other indexes was investigated. According to some scholars $(7,8)$, angiogenesis and lymph node metastasis have a close relationship, and cancer patients with active angiogenesis are more likely to develop lymph node metastasis. Another study (9) has suggested that tumor angiogenesis can promote the contact of active tumor cells and lymphatic channels, thus allowing more tumor cells to enter the lymphoid system. In the present study, the value of hemodynamic parameters in the diagnosis of lymph node metastasis in patients with lung cancer was investigated. 
Previous studies (10) have shown that ultrasound hemodynamic indexes and the lymph node metastasis of breast cancer have a close relationship. However, the diagnostic value of ultrasound hemodynamic parameters in patients with cervical lymph node metastasis of lung cancer has not been evaluated. Therefore, the value of NSE and ultrasound hemodynamic indexes for the individual and joint detection of the lymph node metastasis of lung cancer was assessed, so as to provide a more suitable scheme for the diagnosis of the patients with the cervical lymph node metastasis of lung cancer.

\section{Patients and methods}

General information. The clinical data of 85 patients with lung cancer, admitted to the Qingdao Municipal Hospital (Group) (Qingdao, China) from January 2015 to December 2016, were retrospectively analyzed. There were 51 male and 34 female patients. The average age of the patients was $49.26 \pm 5.45$ years. According to the results of pathological examination, 47 patients with cervical lymph node metastasis were enrolled in the metastatic group, and 38 patients without lymph node metastasis were enrolled in the non-metastatic group. There was no significant difference in sex, age, BMI, smoking history, pathological type of cancer, family medical history, and the stage of cancer between the two groups $(\mathrm{P}>0.05)$ (Table I).

Inclusion and exclusion criteria. Inclusion criteria: Patients diagnosed with lung cancer by pathology. The metastatic group included patients diagnosed with lymph node metastasis by pathology and patients who underwent ultrasound hemodynamic examination. Exclusion criteria: Patients who had undergone anticancer treatment, such as radiotherapy and chemotherapy; patients with severe hepatic and renal dysfunction; patients with other malignant tumors; patients with communication and cognitive impairment; patients who did not cooperate with the examination. All patients and their families agreed to participate in the research and signed an informed consent form. The study was approved by the Ethics Committee of Qingdao Municipal Hospital (Group).

Treatment method. Three milliliters of fasting venous blood were collected from the patients in the morning after they were admitted to the hospital. Serum was separated via centrifugation at $2,600 \mathrm{xg}$ at $4^{\circ} \mathrm{C}$ for $20 \mathrm{~min}$, after the collection of venous blood. Then, the content of NSE in the serum of patients was detected by electrochemiluminescence. The normal reference range of NSE was 0-16.3 $\mu \mathrm{g} / 1$. Color ultrasound hemodynamic examination was performed on all patients. Voluson 730 color Doppler ultrasonic imaging instrument (GE Healthcare) with a probe frequency of 7-12 $\mathrm{MHz}$ was used. The examined part of the patients' neck was fully exposed and examined by color Doppler flow imaging (CDFI). The blood flow resistance index (RI) and pulsatility index (PI) were measured.

Observation indexes and evaluation criteria. The expression level of NSE in serum and the hemodynamic indexes RI and PI were compared between the two groups. The diagnostic efficacy of NSE, RI, PI, and their combination in lymph node metastasis of lung cancer were analyzed by ROC curve analysis.
Statistical analysis. SPSS 19.0 software (Shanghai Yuchuang Network Technology Co., Ltd.) was used for the statistical analysis of experimental data. Chi-square test was used for counting data. Measurement data were expressed as the mean \pm standard deviation, and t-test was used for their analysis between two groups. The experimental graphs were generated using GraphPad Prism 6 software (GraphPad Software, Inc.). $\mathrm{P}<0.05$ was considered to indicate a statistically significant difference.

\section{Results}

Expression of NSE in serum, and the hemodynamic indexes RI and PI in patients of both groups. In the metastatic group, the serum NSE and the indexes RI and PI were $41.24 \pm 11.34 \mu \mathrm{g} / \mathrm{l}$, $0.81 \pm 0.08$, and $2.02 \pm 0.54$, respectively, while those in the non-metastatic group were $33.56 \pm 12.46 \mu \mathrm{g} / 1,0.65 \pm 0.09$, and $1.23 \pm 0.35$, respectively. NSE expression, RI and PI in the metastatic group were significantly higher than those in the non-metastatic group $(\mathrm{P}<0.05)$ (Table II).

Diagnostic value of NSE, RI and PI indexes in cervical lymph node metastasis of lung cancer. The sensitivity and specificity of NSE in the diagnosis of cervical lymph node metastasis of lung cancer were 73.68 and $72.34 \%$, respectively, the diagnostic AUC was 0.783, and the diagnostic critical value was $38.58 \mu \mathrm{g} / 1$. The sensitivity and specificity of RI in the diagnosis of cervical lymph node metastasis of lung cancer were 78.95 and $80.85 \%$, respectively, the diagnostic AUC was 0.820 , and the diagnostic critical value was 0.745 . The sensitivity and specificity of PI in the diagnosis of cervical lymph node metastasis of lung cancer was 81.58 and $68.09 \%$, respectively, the diagnostic AUC was 0.844, and the diagnostic critical value was 1.564 . There was no significant difference in sensitivity and AUC between NSE, RI, and PI in detecting cervical lymph node metastasis of lung cancer (Table III and Fig. 1).

Diagnostic value of NSE combined with RI or PI, and their combination in cervical lymph node metastasis of lung cancer. The sensitivity and specificity of NSE combined with RI were 89.47 and $61.70 \%$, respectively, and the diagnostic AUC was 0.881 . The sensitivity and specificity of NSE combined with PI were 92.11 and $74.47 \%$, respectively, and the diagnostic AUC was 0.905 . The sensitivity and specificity of the combination of NSE, RI, and PI were 97.37 and $57.45 \%$, respectively, and the diagnostic AUC was 0.939. The combination of NSE with RI or PI had good diagnostic value, however, the combination of the three had the highest diagnostic value (Table IV and Fig. 2).

\section{Discussion}

As a malignant tumor with the highest mortality in clinical practice, lung cancer has no obvious early symptoms and is easy to be ignored by patients. Most patients have advanced lung cancer or metastasis at the time of lung cancer detection $(11,12)$. Cervical lymph node metastasis is common in lung cancer patients and one of the main causes of death in lung cancer patients (13). Therefore, timely diagnosis and detection of lymph node metastasis in patients with lung cancer have important clinical significance for effective treatment and 
Table I. General information [n (\%)].

\begin{tabular}{|c|c|c|c|c|}
\hline Factors & $\begin{array}{l}\text { Metastatic group } \\
\quad(\mathrm{n}=47)\end{array}$ & $\begin{array}{l}\text { Non-metastatic group } \\
\qquad(\mathrm{n}=38)\end{array}$ & $\chi^{2}$ & P-value \\
\hline Sex & & & 0.008 & 0.929 \\
\hline Male & $28(59.57)$ & $23(60.53)$ & & \\
\hline Female & $19(40.43)$ & $15(39.47)$ & & \\
\hline Age (years) & & & 0.004 & 0.949 \\
\hline$\leq 49$ & $30(63.83)$ & $24(63.16)$ & & \\
\hline$>49$ & $17(36.17)$ & $14(36.84)$ & & \\
\hline $\operatorname{BMI}\left(\mathrm{kg} / \mathrm{m}^{2}\right)$ & & & 0.024 & 0.876 \\
\hline$\leq 22$ & $28(59.57)$ & $22(57.89)$ & & \\
\hline$>22$ & $19(40.43)$ & $16(42.11)$ & & \\
\hline Smoking history & & & 0.007 & 0.935 \\
\hline Yes & 35 (74.47) & $28(73.68)$ & & \\
\hline No & $12(25.53)$ & $10(26.32)$ & & \\
\hline Pathological type & & & 0.053 & 0.974 \\
\hline Lung adenocarcinoma & $13(27.66)$ & $10(26.32)$ & & \\
\hline Large cell carcinoma & $25(53.19)$ & $20(52.63)$ & & \\
\hline Squamous cell carcinoma & $9(19.15)$ & $8(21.05)$ & & \\
\hline Family medical history & & & 0.010 & 0.922 \\
\hline Yes & $24(51.06)$ & $19(50.00)$ & & \\
\hline No & $23(48.94)$ & $19(50.00)$ & & \\
\hline Stages & & & 0.018 & 0.999 \\
\hline I & $15(31.91)$ & $12(31.58)$ & & \\
\hline II & $16(34.04)$ & $13(34.21)$ & & \\
\hline III & $9(19.15)$ & $7(18.42)$ & & \\
\hline IV & $7(14.89)$ & $6(15.79)$ & & \\
\hline
\end{tabular}

Table II. Expression of serum NSE, and the hemodynamic indexes RI and PI in the two groups.

\begin{tabular}{lcccr}
\hline Factors & Metastatic group $(\mathrm{n}=47)$ & Non-metastatic group $(\mathrm{n}=38)$ & $\mathrm{t}$ & P-value \\
\hline NSE $(\mu \mathrm{g} / \mathrm{l})$ & $41.24 \pm 11.34$ & $33.56 \pm 12.46$ & 2.970 & $<0.050$ \\
RI & $0.81 \pm 0.08$ & $0.68 \pm 0.09$ & 7.043 & $<0.001$ \\
PI & $2.02 \pm 0.54$ & $1.23 \pm 0.35$ & 7.788 & $<0.001$ \\
\hline
\end{tabular}

NSE, neuron-specific enolase; RI, resistance index; PI, pulsatility index.

improvement of survival time of patients with lung cancer. However, the current diagnosis of lymph node metastasis in patients with lung cancer is mainly pathological examination, which is traumatic and may cause pain to the patient (14). The main non-invasive diagnostic method is enhanced CT and PDG-PET, however, they both involve radiation exposure that some patients are unwilling to accept (15). In recent years, with the development of molecular biology, the application of serum tumor markers in the auxiliary diagnosis of lung cancer and the monitoring of recurrence and metastasis has achieved good results $(16,17)$. NSE is recognized as a highly sensitive tumor marker in lung cancer. It is not only expressed in nerve tissue, but also a specific marker of neuroendocrine tumors (18). However, the application of NSE in the diagnosis of cervical lymph node metastasis of lung cancer is relatively rare. Studies (19) have explored the mechanism of lymph node metastasis in lung cancer, suggesting that the condition for lung cancer metastasis is the continuous survival of neovascularization. In the present study, the value of ultrasound hemodynamics in the diagnosis of cervical lymph node metastasis of lung cancer is investigated. Ultrasound hemodynamics is a non-invasive method, which has achieved good results as assistant in the diagnosis of cervical lymph node metastasis of thyroid cancer in recent years. PI and RI, as important indexes of hemodynamics, can effectively reflect the status of blood flow (20). 
A

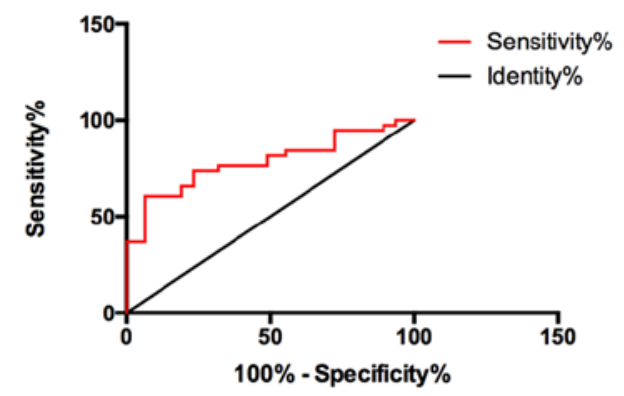

B

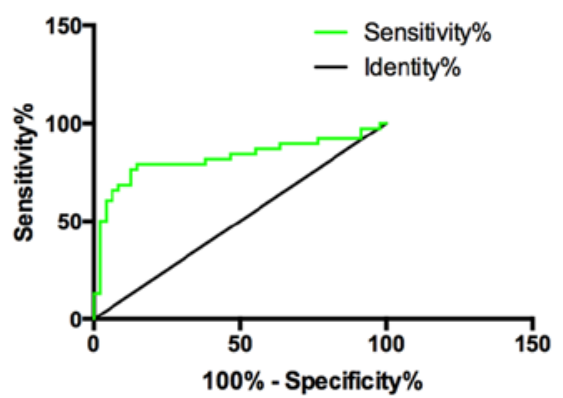

C

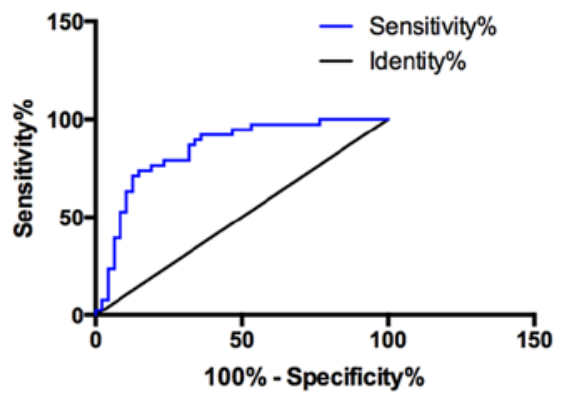

Figure 1. Diagnostic value of NSE, RI, and PI in cervical lymph node metastasis of lung cancer. (A) The sensitivity of NSE in the diagnosis of cervical lymph node metastasis of lung cancer was $73.68 \%$, the specificity was $72.34 \%$, the diagnostic AUC was 0.783 , and the diagnostic threshold was $38.58 \mu \mathrm{g} / 1$. (B) The sensitivity of RI in the diagnosis of cervical lymph node metastasis of lung cancer was $78.95 \%$, the specificity was $80.85 \%$, the diagnostic AUC was 0.820 , and the diagnostic threshold was 0.745 . (C) The sensitivity of PI in the diagnosis of cervical lymph node metastasis was $81.58 \%$, the specificity was $68.09 \%$, the diagnostic AUC was 0.844, and the diagnostic threshold was 1.564. NSE, neuron-specific enolase; RI, resistance index; PI, pulsatility index.

A

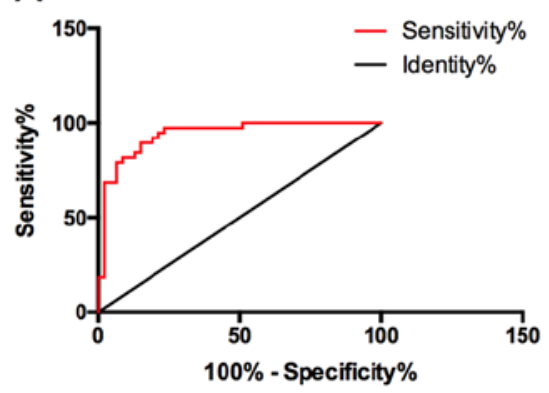

B

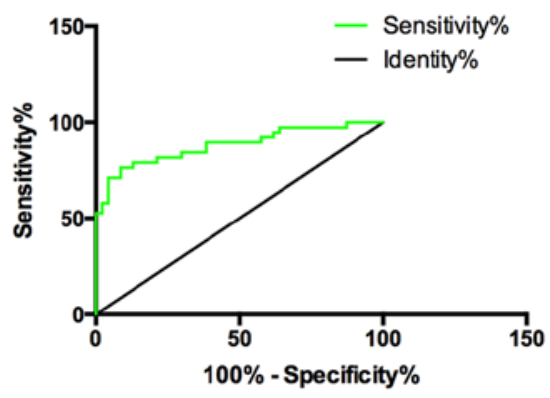

C

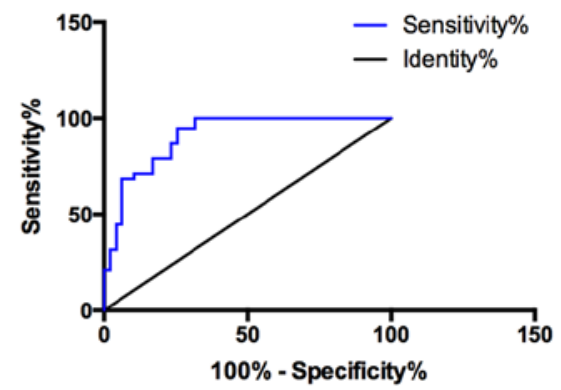

Figure 2. Diagnostic value of NSE combined with RI or PI, and their combination in lymph node metastasis of lung cancer. (A) The sensitivity of NSE combined with RI was $89.47 \%$, the specificity was $61.70 \%$, and the diagnostic AUC was 0.881 . (B) The sensitivity of NSE combined with PI was $92.11 \%$, the specificity was $74.47 \%$, and the diagnostic AUC was 0.905 . (C) The sensitivity of the combination of NSE, RI, and PI in the diagnosis of cervical lymph node metastasis was $97.37 \%$, the specificity was $57.45 \%$, and the diagnostic AUC was 0.939 . NSE, neuron-specific enolase; RI, resistance index; PI, pulsatility index.

Table III. Diagnostic value of NSE, RI, and PI alone, and of their combination in cervical lymph node metastasis of lung cancer.

\begin{tabular}{lccc}
\hline Diagnostic method & Sensitivity (\%) & Specificity (\%) & AUC \\
\hline NSE & 73.68 & 72.34 & 0.783 \\
RI & 78.95 & 80.85 & 0.820 \\
PI & 81.58 & 68.09 & 0.844 \\
\hline
\end{tabular}

NSE, neuron-specific enolase; RI, resistance index; PI, pulsatility index.

Therefore, the value of serum tumor marker NSE and hemodynamic indexes RI and PI individually and jointly in the diagnosis of cervical lymph node metastasis of lung cancer were analyzed. NSE in serum of metastatic and non-metastatic patients was detected by pathology diagnosis. Then, the hemodynamic parameters of the cervical lymph nodes in the two groups were measured by ultrasound. The results revealed that NSE, RI and PI indexes in the metastatic group were significantly higher than those in the non-metastatic group $(\mathrm{P}<0.05)$. These results suggest that a large amount of NSE is secreted by tumor cells when cervical lymph node metastasis occurs in patients with lung cancer. A previous study (21) has explored
Table IV. Diagnostic value of NSE combined with RI or PI, and of their combination in cervical lymph node metastasis of lung cancer.

\begin{tabular}{lccc}
\hline Diagnosis method & Sensitivity (\%) & Specificity (\%) & AUC \\
\hline NSE combined with RI & 89.47 & 61.70 & 0.881 \\
NSE combined with PI & 92.11 & 74.47 & 0.905 \\
NSE, RI, and PI & 97.37 & 57.45 & 0.939 \\
combined diagnosis & & & \\
\hline
\end{tabular}

NSE, neuron-specific enolase; RI, resistance index; PI, pulsatility index.

the relationship between NSE and prognosis of patients with lung cancer, indicating that the expression of NSE in serum of patients with lymph node metastasis is significantly higher than that of patients without metastasis, which is consistent with our conclusion. A previous study (22) has also found that the RI and PI indexes of patients with lymph node metastasis are significantly higher than those of patients without lymph node metastasis when examining the hemodynamics of cervical lymph node metastasis of thyroid cancer. Although this is not a diagnostic study for cervical lymph node metastasis of lung cancer, it also confirms our conclusion. At present, there are 
numerous clinical methods for the diagnosis of lymph node metastasis, such as CT (23), cell biopsy (24) and pathological diagnosis (25). However, there is still radiation in the detection by CT. Both cellular biopsy and pathology are traumatic and can cause more pain to the patient besides the pain caused by the disease. The value of serum tumor marker NSE and the hemodynamic indexes RI and PI individually and jointly were analyzed in the diagnosis of cervical lymph node metastasis of lung cancer. The results showed that there was no significant difference between the sensitivity and AUC of NSE, RI and PI indexes in detecting cervical lymph node metastasis of lung cancer $(\mathrm{P}>0.05)$. However, AUC and the sensitivity of the combination of NSE, RI, and PI were significantly higher than those of NSE, RI, and PI alone $(\mathrm{P}<0.05)$. The specificity of the combined diagnosis was shown to be lower than that of each single diagnosis, which may be a defect of joint diagnosis. These results suggest that NSE, RI, and PI are valuable for the diagnosis of cervical lymph node metastasis of lung cancer, however, their combined diagnosis is more valuable. Up to our knowledge, there are no relevant studies on the diagnostic value of NSE and hemodynamic indexes RI and PI in the diagnosis of cervical lymph node metastasis of lung cancer, and therefore further research it needed.

In conclusion, the serum NSE and hemodynamic indexes RI and PI in patients with cervical lymph node metastasis of lung cancer are higher than those without metastasis, which has certain diagnostic value for cervical lymph node metastasis of lung cancer. However, the diagnostic value of their combination is higher. Thus, it can be used as an auxiliary diagnostic method for cervical lymph node metastasis of lung cancer. In the present study, the combined diagnostic value of more serum tumor markers and hemodynamics was not explored, and there are relatively few reports to confirm our conclusions at present. Therefore, our conclusions still need to be further verified.

\section{Acknowledgements}

Not applicable.

\section{Funding}

No funding was received.

\section{Availability of data and materials}

The datasets used and/or analyzed during the present study are available from the corresponding author on reasonable request.

\section{Authors' contributions}

YK wrote the manuscript. YK and YJ analyzed and interpreted the patient data. YL performed the experiments and designed the study. SB was responsible for the analysis and discussion of the data. All authors read and approved the final manuscript.

\section{Ethics approval and consent to participate}

The study was approved by the Ethics Committee of Qingdao Municipal Hospital (Group) (Qingdao, China). Patients who participated in this research had complete clinical data. Signed informed consents were obtained from the patients or their guardians.

\section{Patient consent for publication}

Not applicable.

\section{Competing interests}

The authors declare that they have no competing interests.

\section{References}

1. Jones JL, Oien KA, Lee JL and Salto-Tellez M: Morphomolecular pathology: Setting the framework for a new generation of pathologists. Br J Cancer 117: 1581-1582, 2017.

2. Braunstein S, Wang L, Newhauser W, Tenenholz T, Rong Y, van der Kogel A, Dominello M, Joiner MC and Burmeister J: Three discipline collaborative radiation therapy (3DCRT) special debate: The United States should build additional proton therapy facilities. J Appl Clin Med Phys 20: 7-12, 2019.

3. Sun W, Yang X, Liu Y, Yuan Y and Lin D: Primary tumor location is a useful predictor for lymph node metastasis and prognosis in lung adenocarcinoma. Clin Lung Cancer 18: e49-e55, 2017.

4. Li N, Tan F, Li J, Shao K, Zhao J, Mu J, Gao S and He J: Blind spot in lung cancer lymph node metastasis: Cross-lobe peripheral lymph node metastasis in early stage patients. Thorac Cancer 9: 480-485, 2018

5. Korkmaz ET, Koksal D, Aksu F, Dikmen ZG, Icen D, Maden E, Onder S, Akbiyik F and Emri S: Triple test with tumor markers CYFRA 21.1, HE4, and ProGRP might contribute to diagnosis and subtyping of lung cancer. Clin Biochem 58: 15-19, 2018.

6. Jiang $\mathrm{ZF}$, Wang $\mathrm{M}$ and $\mathrm{Xu}$ JL: Thymidine kinase 1 combined with CEA, CYFRA21-1 and NSE improved its diagnostic value for lung cancer. Life Sci 194: 1-6, 2018.

7. Liu Y, Ren W, Bai Y, Wan L, Sun X, Liu Y, Xiong W, Zhang YY and Zhou L: Oxyresveratrol prevents murine $\mathrm{H} 22$ hepatocellular carcinoma growth and lymph node metastasis via inhibiting tumor angiogenesis and lymphangiogenesis. J Nat Med 72: 481-492, 2018.

8. Pana ZD, Roilides E, Warris A, Groll AH and Zaoutis T: Epidemiology of invasive fungal disease in children. J Pediatric Infect Dis Soc 6 (Suppl 1): S3-S11, 2017.

9. Pastushenko I, Van den Eynden GG, Vicente-Arregui S, Prieto-Torres L, Alvarez-Alegret R, Querol I, Dirix LY, Carapeto FJ, Vermeulen PB and Van Laere SJ: Increased angiogenesis and lymphangiogenesis in metastatic sentinel lymph nodes is associated with nonsentinel lymph node involvement and distant metastasis in patients with melanoma. Am J Dermatopathol 38: 338-346, 2016.

10. Wu X, Takekoshi T, Sullivan A and Hwang ST: Inflammation and tumor microenvironment in lymph node metastasis. Cancers (Basel) 3: 927-944, 2011.

11. Fitzmaurice C, Dicker D, Pain A, Hamavid H, Moradi-Lakeh M, MacIntyre MF, Allen C, Hansen G, Woodbrook R, Wolfe C, et al; Global burden of disease cancer collaboration: The global burden of cancer 2013. JAMA Oncol 1: 505-527, 2015.

12. Vlachogeorgos GS, Manali ED, Blana E, Legaki S, Karagiannidis N, Polychronopoulos VS and Roussos C: Placental isoform glutathione $\mathrm{S}$-transferase and P-glycoprotein expression in advanced nonsmall cell lung cancer: Association with response to treatment and survival. Cancer 114: 519-526, 2008.

13. Padera TP, Meijer EF and Munn LL: The lymphatic system in disease processes and cancer progression. Annu Rev Biomed Eng 18: 125-158, 2016.

14. Lee DH, Yoon TM, Lee JK and Lim SC: Supraclavicular lymph node excision biopsy in patients with suspected supraclavicular lymph node metastasis of lung cancer: Experience in a Tertiary Hospital. Chonnam Med J 53: 69-72, 2017.

15. Pak K, Kim K, Kim MH, Eom JS, Lee MK, Cho JS, Kim YS, Kim BS, Kim SJ and Kim IJ: A decision tree model for predicting mediastinal lymph node metastasis in non-small cell lung cancer with F-18 FDG PET/CT. PLoS One 13: e0193403, 2018 . 
16. Zhang Q, Wang L, Huang D, Xu M, Weng W, Ni S, Tan C and Sheng W: Pathological risk factors for lymph node metastasis in patients with submucosal invasive colorectal carcinoma. Cancer Manag Res 11: 1107-1114, 2019.

17. Chen C, Wang Y, Fu S, Pan X, Yang J and Wang R: The impact on mediastinal recurrence based on the number of harvested mediastinal lymph nodes and assessed N2 Stations in patients with stage I invasive lung adenocarcinoma. J Thorac Dis 10: 6803-6810, 2018.

18. Shirasawa M, Fukui T, Kusuhara S, Hiyoshi Y, Ishihara M, Kasajima M, Nakahara Y, Otani S, Igawa S, et al: Prognostic significance of the 8th edition of the TNM classification for patients with extensive disease small cell lung cancer. Cancer Manag Res 10: 6039-6047, 2018.

19. Takahashi Y, Suzuki S, Matsutani N and Kawamura M: 18 F-fluorodeoxyglucose positron emission tomography/computed tomography in the evaluation of clinically node-negative non-small cell lung cancer. Thorac Cancer 10: 413-420, 2019.

20. Hwang HS and Orloff LA: Efficacy of preoperative neck ultrasound in the detection of cervical lymph node metastasis from thyroid cancer. Laryngoscope 121: 487-491, 2011.

21. Schneider J, Velcovsky HG, Morr H, Katz N, Neu K and Eigenbrodt E: Comparison of the tumor markers tumor M2-PK, CEA, CYFRA 21-1, NSE and SCC in the diagnosis of lung cancer. Anticancer Res 20: 5053-5058, 2000
22. Shin SH, Kim HS, Jung SH, Xu HD, Jeong YB and Chung YJ: Implication of leucyl-tRNA synthetase 1(LARS1) over-expression in growth and migration of lung cancer cells detected by siRNA targeted knock-down analysis. Exp Mol Med 40: 229-236, 2008.

23. Shao T, Yu L, Li Y and Chen M: Density and SUV ratios from $\mathrm{PET} / \mathrm{CT}$ in the detection of mediastinal lymph node metastasis in non-small cell lung cancer. Zhongguo Fei Ai Za Zhi 18: 155-160, 2015 (In Chinese).

24. Jiang $\mathrm{C}$, Chen Y, Zhu Y and Xu Y: Systematic review and meta-analysis of the accuracy of 18F-FDG PET/CT for detection of regional lymph node metastasis in esophageal squamous cell carcinoma. J Thorac Dis 10: 6066-6076, 2018.

25. Kendirlinan R, Ozkan G, Bayram M, Bakan ND, Tutar M, Gür A and Camsari G: Ultrasound guided fine-needle aspiration biopsy of metastases in nonpalpable supraclavicular lymph nodes in lung cancer patients. Multidiscip Respir Med 6: 220-225, 2011.

This work is licensed under a Creative Commons Attribution-NonCommercial-NoDerivatives 4.0 International (CC BY-NC-ND 4.0) License. 